(65\%) both medication and behavioral therapy. Various medications were prescribed, including clomipramine (59\%), fluoxetine (50\%), and lithium (15\%). At follow-up, 32 (51\%) were still receiving some therapy, and 27 (49\%) were untreated. Total Hairpulling Scale scores showed significant improvements in $52 \%$, and no response in $48 \%$. Improvements had also occurred in measurements of self-esteem, psychosocial functioning, depression and anxiety. Responders had higher baseline depression scores than nonresponders, but a cause and effect correlation was undetermined. None of these subjects had ADHD and none received stimulant therapy.

\title{
BUSPIRONE IN ADHD
}

Buspirone monotherapy $(0.5 \mathrm{mg} / \mathrm{kg}$ daily for 6 weeks) was evaluated in 12 children (10 boys, 2 girls), aged 6 to 12 years, with ADHD at the Maudsley Hospital, London, UK. Compared to baseline scores on Conners Parent and other rating scales, all 12 patients showed significant improvements in hyperacti ity, impulsivity, inattention, and disruptive behavior. Behaviors resumed on discontinuing Buspirone after 6 weeks. Mild dizziness occurred in 2 in the first week. (Malhotra S, Santosh PJ. An open clinical trial of buspirone in children with attention-deficit/hyperactivity disorder. I Am Acad Child Adolesc Psychiatry April 1998;37:364-371). (Reprints: Dr Santosh, Child and Adoilescent Psychiatry, Maudsley Hospital, Denmark Hill, London SE5 8AZ, England).

COMMENT. Controlled trials of buspirone in larger samples of ADHD children seem justified. Buspirone is reported to increase noradrener ic and dopaminergic output, while modulating the serotonergic system. Used previously in children with anxiety, buspirone was tried in ADHD because of low toxicity and twice-daily regimen, not interfering with school schedules. All four dimensions of attention, hyperactivity, impulsivity, and behavioral problems improved, without rebound worsening of symptoms or development of tolerance. Effects on cognitive function need to be addressed, using psychological testing and teacher rating scales.

\section{THE MOZART SONATA AND SPATIAL-TEMPORAL REASONING}

The positive effect of music on spatial-temporal reasoning has been

demonstrated in college students using coherence analysis of electroencephalographic (EEG) recordings at the Center for Neurobiology of Learning and Memory and Department of Physics, University of California, Irvine CA. Right frontal and left temporo-parietal coherent EEG activity was induced by listening to 10 minutes of Mozart (Sonata for Two Pianos in D Major), and enhancement of spatial-temporal reasoning was carried over in 3 of 7 subjects. The long-lasting coherent EEG pattern might indicate structured sequences occurring in cortical function and extending over minutes. (Sarnthein J, vonStein A, Rappelsberger P et al. Pe sistent patterns of brain activity: An EEG coherence study of the positive effect of music on spatial-temporal reasoning. Neurol Res April 1998;19:107-116). (Reprints: Dr Gordon Shaw, Center for Neurobiology of Learning and Memory and Departemt of Physics, University of California, Irvine CA 92697).

COMMENT. Spatial-temporal reasoning is required for chess, mathematics and music appreciation. Exposure to music, specifically Mozart, has been found to enhance cortical activity used in spatial-temporal reasoning, whereas relaxation tapes and minimalist music had no effect. EEG coherence correlates to complex higher brain function, and patterns of cortical activity induced by listening to Mozart carry over to tasks involving spatial-temporal reasoning. 